\title{
Removing artifacts from EEG signal using wavelet transform and conventional filters
}

\author{
MERYEM FELJA, ASMAE BENCHEQROUNE, MOHAMMED KARIM, GHITA BENNIS \\ LIMAS Laboratory, Faculty of Sciences Dhar El Mahraz, Sidi Mohammed Ben Abdellah University
}

\begin{abstract}
EEG) is a signal of an electrical nature reflecting the neuronal activities of the brain. It is used for the diagnosis of certain cerebral pathologies. However, it becomes more difficult to identify and analyze it when it is corrupted by artifacts of non-cerebral origin such as eye movements, cardiac activities ..., therefore, it is essential to remove these parasitic signals. In literature, there are different techniques for removing artifacts. This paper proposes and discusses a new EEG de-noising technique, based on a combination of wavelet transforms and conventional filters. The experimental results demonstrate that the proposed approach can be an effective tool for removing artifact without suppression of any signal components.
\end{abstract}

Key-Words: - EEG signal, artifacts, de-noising, wavelet transform, conventional filters

Received: June 4, 2020. Revised: November 16, 2020. Accepted: December 14, 2020. Published: December 26, 2020.

\section{Introduction}

The electroencephalogram (EEG), represents the electrical activity of the brain, it is very useful for clinical diagnosis and for electro-physiological analysis of the brain [1], [2], [3]. The neuron is the basic functional unit in the brain, it is located in the cerebral cortex, which is divided into four main parts (frontal, parietal, temporal and occipital) each one is responsible of various functions, such as visual information which is processed by the occipital lobe and auditory perception which is processed by the temporal lobe.

EEG activity is recorded from a system of electrodes placed on the head (scalp) [4], [5], giving the cervical rhythm which is the sum of the electrical activity of thousands of neurons.

These recordings are classified into five categories according to their frequencies: frequencies below $0.5 \mathrm{~Hz}$ are considered to be baseline noise; the delta rhythm appears in the frequency domain $(0.5$

to $4 \mathrm{~Hz}$ ) only during deep sleep or in infants as an irregular activity; while the theta rhythm $(4 a ̀$ $7 \mathrm{~Hz}$ ) is a sign of drowsiness and early sleep; the third category is that of the typical rhythm in the resting state with the eyes closed, which is called the alpha rhythm ( 8 to $12 \mathrm{~Hz}$ ); the beta rhythm which is localized in the frequency domain $(13$ to $30 \mathrm{~Hz})$ is remarkable in stressful situations; and finally the gamma rhythm $(>30 \mathrm{~Hz})$ which is associated to the high order functions of the brain[6]. There are also other transient components of EEG that are encountered during sleep, such as the K complex, the mu rhythm which is caused by movement or the stroke component (epilepsy). The specific components which are a response to an external excitation (the evoked potentials) give a different type of EEG recording [7], [8].

These rhythms are used by neurologists to detect and identify cerebral pathologies. However, in the presence of certain artifacts; it becomes difficult to analyze this signal, so it is necessary and essential to remove these sources of noise to ensure correct diagnosis of lot of pathologies. The approach discussed in this paper is divided into two stages: The first step is dedicated to remove unwanted frequencies through the use of conventional filters, while the second step is used to cancel artifacts via the use of an adequate process based on wavelet transform.

\section{Related work}

In recent years, many approaches have been proposed to remove or attenuate artifacts from recorded EEG. Principal component analysis (PCA) has been used to remove artifacts from EEG 
signal [10], [11]. However PCA cannot remove completely artifacts from the signal because the waveforms of some artifacts as ocular artifacts are smaller in amplitude with respect to the ongoing EEG [34], [35]. Later, independent component analysis (ICA) was proposed. The ICA is developed with respect to blind source separation especially ocular artifacts (OA) which is a major source of artifacts that is difficult to remove [6], [8], [15], [16], However, to eliminate this kind of artifacts, ICA needs a reference signal that requires a tedious visual classification of the components [9], [10]. Some research methods have used the modeling of OAs components based on improved support vector machine techniques [14] to isolate them from the EEG [12], [13].

The late of 1980s a novel method was proposed to carry out time-scale analysis of a signal which is the

Wavelet Transforms (WT) [17], [18], [28]. The WT gives a unique framework for many techniques that have been developed for various applications. As WT is suitable for analysis of non-stationary signals which constitute a strong point over spectral analysis, it is appropriate to locate all transient events, which may appear while epileptic seizures [27].

Some researchers have suggested a method based on (WT) and (ICA) to give rise to a new technique: the analysis of independent components of wavelets (WICA). This approach has given satisfactory results in separating noise from brain waves with minimal information loss [19].

\section{Methodology}

The EEG signal has been downloaded from [33] to test our proposed method. The data is sampled at a rate of 128 samples per second. The proposed method consists of removing different kinds of artifacts, as shown in figure 1 our system is composed of two stages:

The first stage devoted to remove the effect of AC line and unwanted frequencies.

The second stage used to remove different kind of noise, whether it's an extrinsic noise or artifacts.

The stage one is used to prepare the EEG signal and pinpointing the useful frequencies where the stage two used to de-noise the prepared EEG signal.

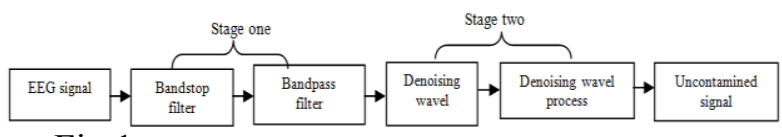

Fig. 1 the proposed system for noise removing to the EEG signal

\subsection{Conventional filters}

The unwanted frequencies that cover the EEG signal are being rejected using notch filter and band pass filters. The noise caused by $\mathrm{AC}$ line is going to be removed via notch filter, while the band pass filters is going to keep just useful frequencies which vary between $0.1 \mathrm{~Hz}$ and $63 \mathrm{~Hz}$.

The concept of digital filters resort to the mathematical approach that we applied to discrete representations of continuous signals for rejecting or reducing undesirable frequencies. In general, the discrete filtering of EEG signals consists in a crossmultiplying of each noisy data point and other neighboring points around it with a set of weights. A recurring cross-multiplication process for each point is likely to remove some artifacts as well as the Direct Current line DC.

These conventional filters algorithms are designed for reducing the start-up and the transient termination through a change in the initial values corresponding to the Direct Current component of the signal component DC.

\subsection{Wavelet Transform (WT)}

The wavelet transform is a spectral estimation method which consists in expressing a general function as an infinite series of wavelets [20], [21]. The main idea behind wavelet analysis is to express a signal as being a linear combination of a particular set of functions called a wavelet transform, which we get by shifting and expanding a single function called the mother wavelet. The decomposition of the signal produces a set of coefficients called wavelet coefficients. Thus, we can reconstruct the signal as a linear combination of the wavelet functions weighted by the wavelet coefficients.

To have an exact and precise reconstruction of the signal, an appropriate number of coefficients must be calculated. The main advantage of wavelets lies in the time-frequency localization [24-26], which implies that the major part of the energy of the wavelet is indeed limited to a precise time interval. The frequency localization shows that the Fourier transform (TF) is band limited. Compared to Short Time Fourier Transform (STFT), the principal advantage of time-frequency localization is the ability to vary the time-frequency aspect ratio, thus giving good frequency localization at low frequencies (long windows) as well as good temporal localization at high frequencies (short time windows). This leads to segmentation, or a tiling of the time-frequency plane which agrees with most physical signals, and precisely those having a 
transient nature. This approach applied to the EEG signal allows revealing all characteristics related to the transient nature of the signal which are not transmitted by the Fourier Transform.

As already indicated in the previous paragraph the wavelet transform (WT) allows to introduce a good representation of a function in the time-frequency domain fundamentally, a wavelet is a function $\Psi \in$ $L^{2}(R) \quad$ with zero average

$$
\int_{-\infty}^{+\infty} \Psi(t) d t=0
$$

The Continuous Wavelet Transformation (CWT) of a signal $\mathrm{x} \square \mathrm{t} \square \square \square$ defined as:

$$
C W T_{\Psi} x(a, b)=\frac{1}{\sqrt{|a|}} \int_{-\infty}^{+\infty} x(t) * \Psi\left(\frac{t-b}{a}\right) d t
$$

Where the function $\Psi(\mathrm{t})$ is the mother wavelet, while the asterisk refers to the complex conjugate, the scalars $a$ and $b(a, b \in R)$ are scaling parameters (dilation and translation), respectively. The scale parameter a defines the oscillation frequency and the wavelet length, and the translation parameter $b$ defines its shifting position.

The application of WT in engineering fields generally requires discrete WT (DWT). DWT is established using discrete values of the scaling parameter $\mathrm{a}=\mathrm{a}_{0}^{\mathrm{m}}$ and the translation parameter $\mathrm{b}=$ $\mathrm{nb}_{0} \mathrm{a}_{0}^{\mathrm{m}}$ then we get:

$\Psi_{(\mathrm{m}, \mathrm{n})}(\mathrm{t})=\mathrm{a}_{0}^{-\mathrm{m} / 2} \Psi\left(\mathrm{a}_{0}^{-\mathrm{m}_{\mathrm{t}}}-\mathrm{nb}_{0}\right)$ with $\mathrm{m}, \mathrm{n} \in \mathrm{Z}, \mathrm{m}$ determines the frequency localization and $n$ determines the temporal localization [23]. In general, we can choose $\mathrm{a}_{0}=2$ and $\mathrm{b}_{0}=1$. This choice allows to define a dyadic-orthonormal WT and also allows to provide the basis of a multiresolution analysis (MRA). So, we can decompose any time series $x(t)$ in terms of approximations provided by the scaling functions $\emptyset_{\mathrm{m}}(\mathrm{t})$ and the details provided by the wavelets $\Psi_{m}(\mathrm{t})[22]$. The wavelet function is associated with the high pass filters (HPF) and the scaling function is associated with the low pass filters (LPF) The decomposition procedure begins by passing the signal through these filters. The approximations are the low frequency components of the time series while the details are the high frequency components. The signal is passed through an HPF and an LPF. Subsequently, the outputs of these two filters are decimated by 2 in order to obtain the detail coefficients and the approximation coefficients at level 1 (A1 and D1). These approximation coefficients are then sent to the second step for the purpose of repeating the procedure. Finally, the signal is decomposed to the desired level.

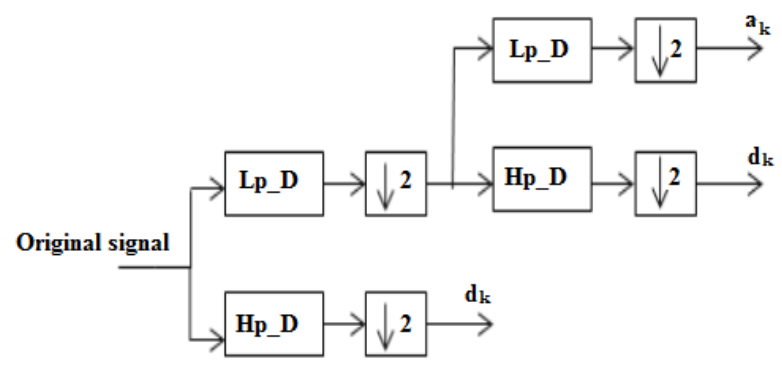

Fig.2. DWT decomposition at several levels (Hp_D and Lp_D refer to high and low pass filters respectively).

\subsection{Wavelet families}

The wavelength families are grouped in the

\begin{tabular}{|c|c|}
\hline $\begin{array}{l}\text { Wavelets } \\
\text { Families }\end{array}$ & Wavelets \\
\hline Daubechies & 'db'or'haar','db2', ..,'db10',..,'db10', ,.,'db45' \\
\hline Coiflets & 'coifl', ...,'coif5' \\
\hline Symlets & 'sym2', ..,'sym8', ..,'sym45' \\
\hline $\begin{array}{l}\text { Discrete } \\
\text { Meyer }\end{array}$ & ‘dmey’ \\
\hline Biorthogonal & $\begin{array}{l}\text { 'bior1.1','bior1.3','bior1.5','bior2.2',',bior2.4','b } \\
\text { ior2.6','bior2.8','bior3.1','bior3.3','bior3.5','bio } \\
\text { r3.7','bior3.9','bior4.4','bior5.5','bior6.8'. }\end{array}$ \\
\hline
\end{tabular}
following table with regard to wavelets.

Tab.1. wavelet families

- Daubachies

In general, the wavelets of the Daubechies family are known by the sign $\mathrm{dbN}$ ( $\mathrm{N}$ is the order). They belong to orthogonal wavelets.

\section{- Coiflets}

It is a discrete wavelet designed by Ingrid Daubechies in order to have a scaling function with moments of disappearance. The scaling function and the wavelet function needs to be normalized by a common factor.

\section{- Symlets}

The wavelets of the symlet family are known by symN ( $\mathrm{N}$ isorder). These wavelets are almost symmetrical, orthogonal and biorthogonal, they are also suggested by Daubechies as being a modification of the $\mathrm{db}$ family. 
The characteristics of these two wavelet families are similar [19].

\section{- Biorthogonal}

Biorthogonal filters state a superset of orthogonal wavelet filters and have found their use virtually in all fields where wavelets are widely used. The biorthogonal family wavelets are known as bior. biorthogonal wavelet transformation are widely used in image processing, since it makes multiresolution analysis possible and does not produce redundant information.

\subsection{Discrete wavelet de-noising approach}

To remove certain artifacts such as eye movements, blinking eyes, muscular activities we introduced the technique of wavelet de-noising, this process essentially consists of two main stages:

Step1: is used to decompose the EEG signal so as to obtain the detail and approximation coefficients, via a set of conventional filters: low pass filters and high pass filters. In fact, the low pass filter makes it possible to extract the coefficients of detail, while the high pass filter serves to obtain the approximation coefficients [5].

Step 2: is used to find the threshold value of the two $6]$.

To analyze the EEG signal, Symlet5 was used as a basic function, decomposed into 7 levels.

\subsection{Evaluation criteria}

To evaluate the performance of our proposed method; we used 3 criteria, which are Signal-toNoise Ratio SNR (1), Mean Squared Error MSE(2), and Cross Correlation Function CCF (3)

$$
\begin{aligned}
& S N R=10 \log \left|\frac{\sum_{n} \hat{x}^{2}(n)}{\sum_{n}[x(n)-\hat{x}(n)]^{2}}\right| \\
& M S E=\frac{1}{N} \sum_{n=1}^{N}[x(n)-\hat{x}(n)]^{2} \\
& C C F_{x \hat{x}}(m)=\left\{\begin{array}{cc}
\sum_{n=0}^{N-m-1} x_{n+m} x_{n}^{*} & m \geq 0 \\
C C F_{x \hat{x}} & m<0
\end{array}\right.
\end{aligned}
$$

With $\mathrm{x}(\mathrm{n})$ is the original signal while $\mathrm{x}(\mathrm{n})$ is the filtered signal, the sign *refers to the complex conjugate. The best performances of our method when we achieve the highest value of the SNR, the lower value of the MSE and also, the highest value of the points of intersection of CCF.

\section{Results and discussion}

Since there are no real recordings of EEG signals, these recordings have been downloaded from [33] to test our proposed method. The data is sampled at a rate of 128 samples per second.

Figure 3 shows two noisy EEG signal recording channels in which we try to remove all types of existing noises.

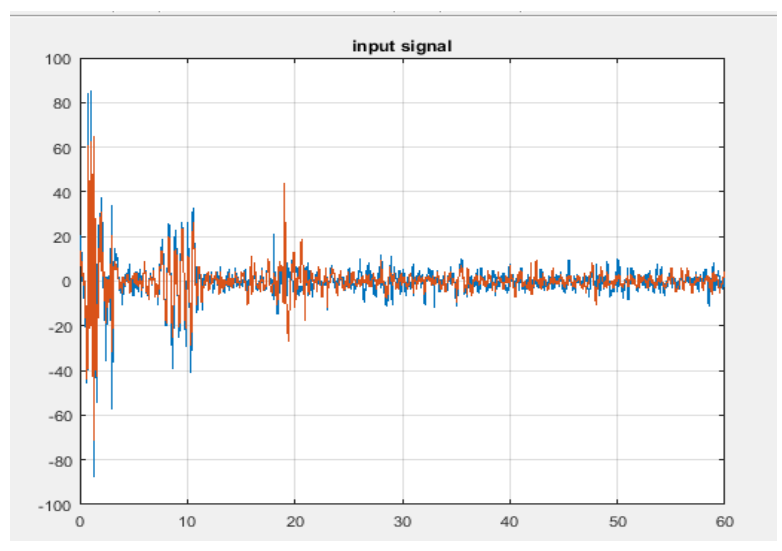

Fig.3. Two channels of input signal EEG

\subsection{Notch and band pass filters}

In the first step, the two filters were used: the band pass filter and the band stop filter.

Indeed, the band stop filter has the role of cancelling the undesirable effects of the AC line and all its harmonics (49-51) Hz that overlap with the EEG signal especially with gamma band, which causes an increase of the amplitude of its frequencies. While the band-pass filter is used to eliminate certain artifacts such as ocular artifacts, and it is also effective in removing the DC line produced by the electronic equipment. The following figure shows the two channels of filtered EEG signals of all of its noise types reported above

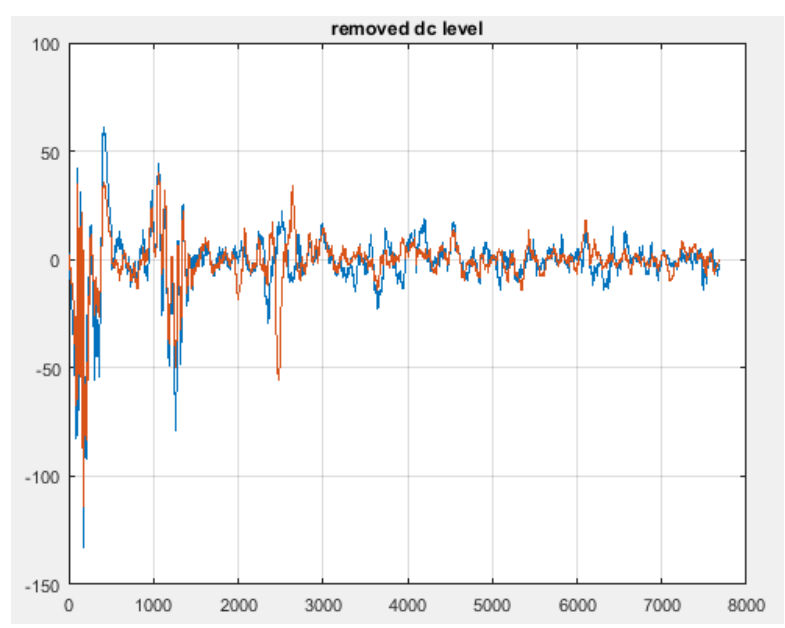

Fig.4. two channels of EEG signal filtred by notch filter and band pass filt 


\subsection{Wavelet de-noising process}

In the second stage, the system suppresses all kind of artifacts that cover the recorded signal with wavelet process. The wavelet transform is extremely powerful for representing different features of signals like trends, repeated patterns, discontinuities, and; it is particularly effective for non-stationary signal especially in our case where the EEG signal represents all these events. Therefore, the wavelet transform is an efficient and robust tool to properly analyze signals of a transient nature since we can get the information about time and frequency

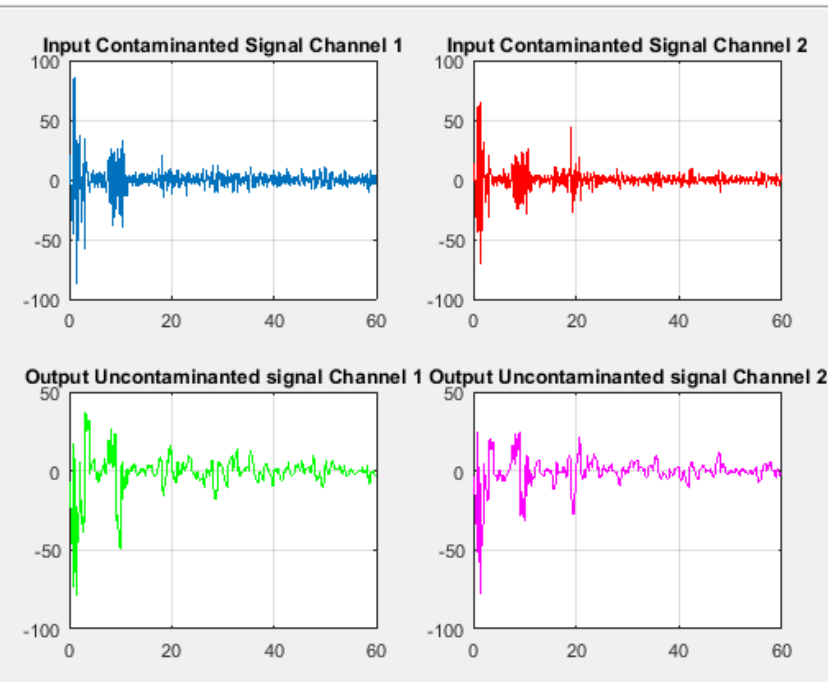

Fig.5. Two channels of EEG signal recording denoised by bandstop filter, bandpass filter and wavelet transform

From the figure above, we notice that the majority of artifacts that affect the EEG signal are removed without affecting the components of the EEG signal. We measured the performance of this stage by calculating SNR, MSE, and CCF at the output of the wavelet denoising technique used for two different signals

\begin{tabular}{|l|l|l|l|l|l|l|}
\hline $\begin{array}{l}\text { Subjec } \\
\text { ts }\end{array}$ & \multicolumn{2}{|c|}{ SNR(dB) } & \multicolumn{2}{c|}{ CCF } & \multicolumn{2}{c|}{ MSE } \\
\hline & $\begin{array}{l}\text { Chann } \\
\text { el 1 }\end{array}$ & $\begin{array}{l}\text { Chann } \\
\text { el 2 }\end{array}$ & $\begin{array}{l}\text { Chann } \\
\text { el 1 }\end{array}$ & $\begin{array}{l}\text { Chann } \\
\text { el 2 }\end{array}$ & $\begin{array}{l}\text { Chann } \\
\text { el 1 }\end{array}$ & $\begin{array}{l}\text { Chann } \\
\text { el 2 }\end{array}$ \\
\hline 1 & $\begin{array}{l}56.777 \\
2\end{array}$ & 62.589 & 0.8835 & 0.8753 & 0.3696 & 0.2124 \\
\hline 2 & $\begin{array}{l}49.423 \\
8\end{array}$ & $\begin{array}{l}51.388 \\
4\end{array}$ & 0.7476 & 0.9366 & 0.8671 & 0.6879 \\
\hline
\end{tabular}

Tab.2. The performance of wavelet de-noising process

according to the results of the table above, we can note that the values of the criteria SNR, MSE and CCF vary from a channel to another on the one hand, and from a subject to another on the other hand, and this is due to the kind of artifacts. We observe that the SNR of the EEG signals is high, the
MSE is low for both cases, the CCF gave a good value too, which proves that the de-noised signals remain in correlation with the previous stage, which means the effectiveness of the proposed method.

\section{Conclusion}

The noise present in the electroencephalogram signals is known like artifacts and these artifacts must be suppressed from the original signal for appropriate treatment and analysis of electroencephalogram signals to facilitate the detection of various diseases related to the brain. This work proposes a filtering approach to remove the noise and errors of electroencephalogram signal. This process consists of two stages: conventional Filters (band pass filter and band stop filter), and Wavelet de-noising process. Firstly the band stop filter rejects the effects of AC line (49-51) Hz, while the band pass filter only allows the useful frequencies to pass (0.1-63) Hz. Secondly the wavelet transform offers a perfect success in the rejecting the most of artifacts that overlapped with recorded signals, without affecting the electroencephalogram signal components, we tested the performance of the technique proposed by several criteria, it gave us satisfactory results Finally; this research provides an efficient technique for improving the quality of electroencephalogram signals in biomedical analysis.

\section{References:}

[1] Niedermeyer E, Silva FH (1993) Electroencephalography: Basic principles, clinical applications and related fields. Lippincott, Williams \& Wilkins, Philadelphia. DEFINITION EEEG

[2] Akin, M., Investigation of Excited Brain Potential With Spectral Analysis Methods, Ph.D. Thesis, University of Erciyes, Turkey, 1995.

[3] Arserim, M. A., The Estimation of Brain Signals With Modern Spectral Analysis Methods, Master Thesis, Diyarbakir, Turkey, 2001.

[4] Kolb et Whishaw, 2002] KOLB, B. et WHISHAW, I. (2002). Cerveau et comportement. De Boeck Universite lobe

[5] Westen et Garitte, 2000] WESTEN, D. et GARITTE, C. (2000). Psychologie : Pensée, cerveau et culture. De Boeck Université. lobe

[6] S. Tong and N.V.Thacor, Engineering in Medicine \& Biology-Quantitative EEG 
Analysis Methods and Clinical Applications,Boston/London: Artech House, 2009

[7] R. A. Fisher, "The Use of Multiple Measurements in Taxonomic Problems", Annals of Eugenics, vol. 7, no. 2, pp. 179-188, 1936

[8] G. Blanchet et M. Charbit, Digital Signal and Image Processing using MATLAB. HERMES Science Europe Ltd, 2001.

[9] R. Begg, D. T. H. Lai et M.Palaniswami,Computational Intelligence in Biomedical Engineering.

[10] T. D. Lagerlund, F. W. Sharbrough, and N. E. Busacker, "Spatial filtering of multichannel electroencephalographic recordings through principal component analysis by singular value decomposition," Clin.Neurophysiol.,vol. 14, no. 1, pp. 73-82, 1997.

[11] I. T. Joliffe, Principal Component Analysis. New York, NY, USA: Springer-Verlag, 1986

[12] Walters-Williams J, Li Y (2011) Performance comparison of known ICA algorithms to a wavelet- ICA merger. Signal Process, 5 (3):8092.

[13] R. Vigario, J. Sarela, V. Jousmaki, M. Hamalainen, and E. Oja, "Independent component approach to the analysis of EEG and MEG recordings,'IEEE Trans. Biomed. Eng., vol. 47, no. 5, pp. 589-593, May 2000.

[14] S.-Y. Hao, K.-Q. Shen, C. J. Ong, E. P. V. Wilder-Smith, and X.-P. Li"Automatic EEG artifact removal: A weighted support vector machineapproach with error correction," IEEE Trans. Biomed. Eng., vol. 56, no. 2,pp. 336344, Feb. 2009.

[15] W. Lu and J. C. Rajapakse, "ICA with reference," in Proc. 3rd Int. Conf. Independent Component Analysis Blind Signal Separation, 2001, pp. 120-125

[16] A. Hyv"arinen and E. Oja, "A fast fixed-point algorithm for independent component analysis," Neural Comput., vol. 9, no. 7, pp. 1483-1492, 1997

[17] Mallat, S.G., A theory for multiresolution signal decomposition:The Wavelet representation, IEEE Transaction on Pattern Analysis and Machine Intelligence, (1989), pp.674-693

[18] P. Addison, in: Illustrated Wavelet Transform Handbook, 1st ed., Taylor and Francis, USA, 2002, pp. 7-17. WT

[19] Walters-Williams J, Li Y (2011) Performance comparison of known ICA algorithms to a
wavelet-ICA merger. Signal Process, 5 (3):8092.

[20] S. Mallat, A Wavelet Tour of Signal Processing, San Diego, CA: Academic, 1998

[21] S. G. Mallat, "A theory for multiresolution signal decomposition Thewavelet representation," IEEE Trans. Pattern Anal. Mach. Intell., vol. 11,no. 7, pp. 674693, Jul. 1989.

[22] V. J. Samar, A. Bopardikar, R. Rao, and K. Swartz, "Wavelet analysisof neuroelectric waveforms: A conceptual tutorial," Brain Lang.,vol. 66,no. 1, pp. 760, 1999 DWT LA NON REDONDANCE

[23] I. Daubechies, Ten Lectures on Wavelets, Philadelphia: Society for Industrial and Applied Mathematics, 1992.

[24] H. He and J. A. Starzyk, "A Self-Organizing Learning Array System for Power Quality Classification Based on Wavelet Transform," IEEE Transaction On Power Delivery, vol. 21(1), pp. 286-295, 2006.

[25] S. Avdakovic and A. Nuhanovic, "Identifications and Monitoring of Power System Dynamics Based on the PMUs and Wavelet Technique," International Journal of Energy and Power Engineering, vol. 3, pp.202209, 2010.

[26] Vincent J. Samar, Ajit Bopardikar, Raghuveer Rao and Kenneth Swartz, "Wavelet analysis of neuroelectric waveforms: A conceptual tutorial “, Brain and Language, 66, pp. 7 - 60, 1999

[27] C. Sidney Burrus, Ramesh A Gopinath and Haitao Guo, "Introduction to Wavelets and Wavelet Transforms ", Prentice-Hall international Inc, 1998.

[28] W.J. Tompkins, “ Biomedical digital signal processing", New Jersey, Prentice Hall, 1993

[29] Wachowiak MP, Rash GS, Quesada PM, Desoky AH (2000). Wavelet-based noise removal for biomedical signals: A comparative study. IEEE Transactions on biomedical engineering, vol. 47, no. 3,pp. 360-368.

[30] Hussain M S, Reaz M B I, Mohd-Yasin F, Ibrahimy M I (2009) Electromyography signal analysis using wavelet transform and higher order statistics to determine muscle contraction. Expert Syst., 26(1):35-48.

[31] Ye H, Deng G, Mauger SJ, Hersbach AA, Dawson PW, et al. (2013) A Wavelet-Based Noise Reduction Algorithm and Its Clinical Evaluation in Cochlear Implants. PLoS ONE 8(9): e 75662

[32] Website: https://www.physionet.org/cgibin/atm/ATM 
[33] O. G. Lins, T. W. Picton, P. Berg, and M. Scherg, "Ocular artifacts in EEG and eventrelated potentials, I: Scalp topography," Brain Topography, vol. 6, pp. 51-63, 1993.

[34] O. G. Lins, T. W. Picton, P. Berg, and M. Scherg, "Ocular artifacts in recording EEGs and event-related

\section{Creative Commons Attribution License 4.0 (Attribution 4.0 International, CC BY 4.0)}

This article is published under the terms of the Creative Commons Attribution License 4.0

https://creativecommons.org/licenses/by/4.0/deed.en_US 